\title{
Aspects of nonrelativistic quantum gravity
}

\author{
Johan Hansson* \\ Department of Physics, Luleå University of Technology \\ SE-971 87 Luleå, Sweden \\ (Received on 8 September, 2009)
}

\begin{abstract}
A nonrelativistic approach to quantum gravity is studied. At least for weak gravitational fields it should be a valid approximation. Such an approach can be used to point out problems and prospects inherent in a more exact theory of quantum gravity, yet to be discovered. Nonrelativistic quantum gravity, e.g., shows promise for prohibiting black holes altogether (which would eliminate singularities and also solve the black hole information paradox), gives gravitational radiation even in the spherically symmetric case, and supports non-locality (quantum entanglement). Its predictions should also be testable at length scales well above the "Planck scale", by high-precision experiments feasible with existing technology.
\end{abstract}

Keywords: Quantum gravity; Black holes; Information paradox; Testable

The greatest fundamental challenge facing physics has for many years been to reconcile gravity with quantum physics. There have been numerous attempts to do so, but so far there is no established and experimentally/observationally tested theory of "quantum gravity", the two main contenders presently being string theory [1] and loop quantum gravity [2], with "outsiders" like twistor theory [3], noncommutative geometry [4], etc.

The motivations for studying nonrelativistic quantum gravity, apart from the simple and well-defined mathematics, are:

1) Quantum theory is supposed to be universal, i.e., it should be valid on all length scales and for all objects, as there in principle exists no size/charge/mass-limit to its applicability. In atomic physics the practical restriction comes about because there is a limit to arbitrarily large atomic nuclei as, i) the Coulomb force between protons is repulsive, eventually overpowering the strong nuclear force trying to hold the nucleus together, ii) the additional weak force makes neutron-rich nuclei decay before they grow too large. Also, the electric charge comes in both positive and negative, and as a result a big lump of matter is almost always electrically neutral ${ }^{1}$. Neither of these limitations are present in "pure" quantum gravity.

2) For weak gravitational fields the nonrelativistic theory should be sufficient. The weak-field newtonian limit is even used for determining the constant $\kappa$ in Einstein's field equations of general relativity $G_{\mu \nu}=\kappa T_{\mu \nu}$. The nonrelativistic limit is also almost always sufficient for practical purposes in non-quantum gravity, except for a handful of extreme cases (notably black holes and the very early universe), although high-precision experiments in, e.g., the solar system can and do show deviations from the nonrelativistic theory, always in favor of general relativity [5].

3) Even for strong gravitational fields the newtonian picture gives the same prediction as general relativity for the Schwarzschild radius of a spherically symmetric, nonrotating black hole, and correct order of magnitude results

\footnotetext{
*Electronic address: c. johan. hansson@ltu. se

1 The same also applies for e.g. the strong force, as the three different color charges ("red", "green", "blue") always combine to produce color-neutral hadrons and bulk matter.
}

for neutron stars and cosmology. This could make it possible to deduce at least qualitative results about strongly coupled quantum gravity, as the nonrelativistic viewpoint should give reliable first order quantum gravitational results.

On the other hand would any "absurd" results obtained from nonrelativistic quantum gravity, deviating from observations, implicate either that:

A) General relativity cannot be quantized ${ }^{2}$. An unsuccessful special case (the weak field limit) would disprove the general case, whereas the opposite is not true.

or

B) Quantum mechanics fails at "macroscopic" distances and for macroscopic objects. This would mean that we in gravity have a unique opportunity to understand the "measurement problem" in quantum mechanics, as proposed by e.g. Károlyházy [7] and Penrose [8]. In that case we can use gravity to probe the transition between quantum $\rightarrow$ classical behavior in detail, i.e. get experimental facts on where, how and when the inherently undecided quantum world of superpositions turns into the familiar objective classical everyday world around us. Fundamental quantum gravity and the quantum mechanical measurement problem may well be intertwined and might need to be resolved simultaneously in a successful approach.

In nonrelativistic quantum gravity, at least as long as the system can be approximately treated as a 2-body problem, it is possible to use the mathematical identity between the electrostatic Coulomb force in the hydrogen atom, and Newton's static gravitational force under the substitution $Z e^{2} / 4 \pi \varepsilon_{0} \rightarrow$ $G m M$. For weak electromagnetic fields, as in the hydrogen atom, the electrodynamic corrections to the static Coulomb field are very small, making the approximation excellent. The same applies to gravity, dynamical effects from general relativity are negligible for weak gravitational fields. A gravitationally bound 2-body system should then exhibit the same type of "spectrum" as a hydrogen atom, but emitted in (unobservable) graviton form instead of photons.

For a free-falling 2-body system, e.g. in a satellite experiment enclosed in a spherical vessel, it should in principle

\footnotetext{
2 This is an automatic consequence of "emergent" gravity, e.g. Sakharov's theory [6], where gravity is a non-fundamental interaction and rather a macroscopic consequence of other forces and fields.
} 
be possible to measure the excitation energies for a suitable system. An analogous result has seemingly already been accomplished for neutrons in the gravitational field of the earth [9], although there are some quantum gravity ambiguities as noted below.

The gravitational "Bohr-radius", $b_{0}$, the innermost radius of circular orbits in the old semi-classical Bohr-model and also the distance $r$ for which the probability density of the Schrödinger equation ground-state peaks, is

$$
b_{0}=\frac{h^{2}}{4 \pi^{2} G m^{2} M}=\frac{\hbar^{2}}{G m^{2} M},
$$

and the quantum-gravitational energy levels

$$
E_{n}(\operatorname{grav})=-\frac{2 \pi^{2} G^{2} m^{3} M^{2}}{h^{2}} \frac{1}{n^{2}}=-\frac{G^{2} m^{3} M^{2}}{2 \hbar^{2}} \frac{1}{n^{2}}=-E_{g} \frac{1}{n^{2}},
$$

where $E_{g}=G^{2} m^{3} M^{2} / 2 \hbar^{2}$ is the energy required to totally free the mass $m$ from $M$ in analogy to the Hydrogen case, whereas the expectation value for the separation is

$$
\langle r\rangle_{g r a v} \simeq n^{2} b_{0}=\frac{n^{2} \hbar^{2}}{G m^{2} M} .
$$

All analytical solutions to the Schrödinger equation, the hydrogen wave-functions, carry over to the gravitational case with the simple substitution $a_{0} \rightarrow b_{0}$.

$$
\psi_{n l m}=R(r) \Theta(\theta) \Phi(\phi)=N_{n l m} R_{n l} Y_{l m},
$$

where $N_{n l m}$ is the normalization constant, $R_{n l}$ the radial wavefunction, and $Y_{l m}$, the spherical harmonics, contain the angular part of the wavefunction.

We notice (e.g. through $b_{0}$ ) that, e.g., the planets in the solar system must be in very highly excited quantum gravitational states. In that sense they are analogous to electrons in "Rydberg atoms" in atomic physics [10].

For excited states with $l \neq 0$, and very large $n$ and $l$, the expectation value of the distance is

$$
\langle r\rangle \simeq \frac{1}{2}\left(3 n^{2}-l^{2}\right) b_{0}
$$

however as that is for an ensemble (average over many measurements), for a single state it is in principle more appropriate to use the most probable radial distance ("radius" of orbital)

$$
\tilde{r}=n^{2} b_{0},
$$

as a measure for the expected separation. However, for $n$ large and $l=l_{\max }=n-1$ the two coincide so that $\langle r\rangle=\tilde{r}$

It is easy to show that for, e.g., Kepler's law to apply, $l$ must be very close to $n$ :

The period of revolution can be written

$$
T=\frac{2 \pi m \tilde{r}^{2}}{L}=\frac{2 \pi m \tilde{r}^{2}}{l \hbar},
$$

and assuming maximality for the angular momentum, $l \simeq n$, gives

$$
T \simeq \frac{2 \pi m \tilde{r}^{2}}{n \hbar}
$$

Solving the most probable distance, Eq. (6), for $n$ gives

$$
n=\frac{m \sqrt{G M \tilde{r}}}{\hbar}
$$

so that

$$
T \simeq \frac{2 \pi \tilde{r}^{3 / 2}}{\sqrt{G M}}
$$

which is Kepler's law. So, the conclusion is that all the planets in the solar system are in maximally allowed angular momentum states quantum mechanically. Even though the maximality of $L$ and $L_{z}$ are automatic in the classical description, it is far from obvious why the same should result from the more fundamental quantum treatment, as noted below.

For states with $l=l_{\max }=n-1$ and $m= \pm l$ : i) There is only one peak, at $r=\tilde{r}$, for the radial probability density, and the "spread" (variance) in the $r$-direction is given by $^{3} \Delta r=\sqrt{\left\langle r^{2}\right\rangle-\langle r\rangle^{2}} \simeq n^{3 / 2} b_{0} / 2$, ii) The angular $\theta$-part of the wavefunction for maximal $m$-quantum number $|m|=l$, is $\propto \sin ^{l} \theta$. The probability density thus goes as $\sin ^{2 l} \theta$ in the $\theta$-direction, meaning that only $\theta=\pi / 2$ is nonvanishing for large $l$. The azimuthal $(\phi)$ part of the angular wavefunction $Y_{l m}$ is purely imaginary, making it drop out of the probability density, so that all values of $\phi$ are equally likely. (This $\phi$-symmetry is a consequence of conservation of angular momentum in a central potential.) The total planetary probability density is thus "doughnut" (torus-like) shaped, narrowly peaking around the classical trajectory.

From a quantum gravity standpoint, the system could be in any and all of the degenerate states, and usually at the same time, so typical for quantum mechanical superposition. Even for given energy and angular momentum there is no reason for objects to be in any particular eigenstate at all of the $2 l+1$ allowed, and certainly not exclusively $m= \pm l$. The radial probability distribution in general has $n-l$ maxima. Thus, only for $l=l_{\max }=n-1$ has it got a unique, highly peaked maximum. The degeneracy for a given $n$ is $n^{2}$. Whenever $l<l_{\max }$, the radial wavefunction is highly oscillatory in $r$ as it has $n-l$ nodes. The same goes for the angular distribution as there in general are $l-m$ nodes in the $\theta$-direction. For a general $R_{n l} Y_{l m}$ objects could be "all over the place", and in simultaneous, co-existing superposed states with different quantum numbers. Consequently, nonrelativistic quantum gravity cannot solve the quantum mechanical measurement problem, possibly because it lacks the non-linear terms conjectured to be needed [8].

To get the innermost allowed physical orbit for any "testparticle", $m$, we must impose the physical restriction that the binding energy cannot exceed the test particle mass-energy

\footnotetext{
${ }^{3}$ The hydrogen wavefunctions for the gravitational case give $\left\langle r^{2}\right\rangle=\left[5 n^{2}+\right.$ $1-3 l(l+1)] n^{2} b_{0}^{2} / 2$ and $\langle r\rangle=\left[3 n^{2}-l(l+1)\right] b_{0} / 2$.
} 
(as the energy of the total system otherwise could not be conserved), thus

$$
E_{g}(\max )=m c^{2}
$$

As $E_{g}$ can be written

$$
E_{g}=\frac{G m M}{2 b_{0}},
$$

we get

$$
b_{0}(\min )=\frac{G M}{2 c^{2}}=\frac{R_{S}}{4},
$$

where $R_{S}=2 G M / c^{2}$ is the Schwarzschild radius. It is amusing to see how close $b_{0}(\mathrm{~min})$ is to $R_{S}$ and one cannot help speculate that a more complete theory of quantum gravity could ensure that $r>R_{S}$ always, and thus forbid black holes altogether ${ }^{4}$. In addition, all non-gravitational radiation mechanisms during collapse have here been neglected. The object $M$ must be put together somehow, but if $r_{\min }>R_{S}$ it can never accrete enough matter to become a black hole, as the infalling mass (energy) instead will be radiated away in its totality (in gravitons), making a black hole state impossible [11]. This would, in an unexpected way, resolve the black hole information loss paradox. Even though $r=R_{S}$ represents no real singularity, as it can be removed by a coordinate transformation, anything moving inside $r<R_{S}$ will, according to classical general relativity, in a (short) finite proper time reach the true singularity at $r=0$. If quantum gravity could ensure that $r>R_{S}$ always, gravity would be singularity free.

Let us briefly look at radiative transitions. From the dipole approximation in atomic physics an elementary quantum (photon) transition requires $\Delta l= \pm 1$. A quadrupole (graviton) approximation in quantum gravity instead requires $\Delta l= \pm 2$. So, a typical elementary energy transfer in a highly excited, gravitationally bound 2-body quantum gravitational system is

$$
\Delta E=-E_{g}\left(n^{-2}-(n-2)^{-2}\right) \simeq \frac{4 E_{g}}{n^{3}} .
$$

We also see that the gravitational force is not really conservative, even in the static newtonian approximation. The changes in kinetic and potential energies do not exactly balance, $\Delta K \neq \Delta U$, the difference being carried away by gravitons in steps of $\Delta l=2$. Also, in the quantum gravity case there is gravitational radiation even in the spherically symmetric case, which is forbidden in classical general relativity.

We are now equipped to return to the experiment with neutrons in the gravitational field of the earth [9], claiming to have seen, for the first time, quantum gravitational states in the potential well formed by the approximately linear gravitational potential near the earth surface and a horizontal neutron mirror. An adjustable vertical gap between the mirror

\footnotetext{
${ }^{4}$ For the hydrogen atom the corresponding value is $a_{0}(\min ) \simeq 1.4 \times 10^{-15}$ $\mathrm{m}$, or one-half the "classical electron radius", whereas $R_{S} \simeq 10^{-53} \mathrm{~m}$, so that $a_{0}(\min ) \gg R_{S}$. But we implicitly already knew that. The Coulomb force does not turn atoms into black holes.
}

and a parallel neutron absorber above was found to be nontransparent for traversing neutrons for separations less than $\sim 15 \mu \mathrm{m}$ (essentially due to the fact that the neutron ground state wavefunction then overlaps the absorber). As the neutron in such a well, from solving the Schrödinger equation, has a ground state wavefunction peaking at $\sim 10 \mu \mathrm{m}$, with a corresponding energy of $\simeq 1.4 \times 10^{-12} \mathrm{eV}$, the experimental result is interpreted to implicitly having verified, for the first time, a gravitational quantum state.

If we instead analyze the experiment in the framework of the present article, the same experimental setup gives $b_{0} \simeq 9.5 \times 10^{-30} \mathrm{~m}, E_{g} \simeq 2.2 \times 10^{35} \mathrm{eV}$. Close to the earth's surface, $\tilde{r} \simeq R_{\oplus} \simeq 6.4 \times 10^{6} \mathrm{~m}$, the radius of the earth, giving $n \simeq 8.2 \times 10^{17}$, resulting in a typical energy for an elementary quantum gravity transition $\Delta E \simeq 4 E_{g} / n^{3} \simeq 1.6 \times 10^{-18}$ $\mathrm{eV}$. For a cavity of $\Delta \tilde{r}=15 \mu \mathrm{m}$, and $n \gg \Delta n \gg 1$, one gets $\Delta E=E_{g} b_{0} \Delta \tilde{r} / \tilde{r}^{2} \simeq 0.7 \times 10^{-12} \mathrm{eV}=0.7 \mathrm{peV}$, to be compared to the value $1.4 \mathrm{peV}$ as quoted in [9]. Even though the present treatment gives a similar value for the required energy, it need not be the result of a single quantum gravity state as calculated in [9], but rather $\leq 10^{6}$ gravitons can be emitted/absorbed. From the treatment in this article it is thus not self-evident to see why the experimental apparatus [9] should be non-transparent to neutrons for vertical separations $\Delta h<15 \mu \mathrm{m}$.

Thus, the difference regarding quantized energy levels for an experiment with neutrons "falling" 5 under the influence of earth's gravity with mirror (as in [9]) or without (above) shows that nonrelativistic quantum gravity is dependent on global boundary conditions, where the boundary in principle can lie arbitrarily far away. This comes as no surprise, as the Schrödinger equation models the gravitational interaction as instantaneous, contrasted with the case in general relativity where the behavior in free-fall only depends on the local properties of mass-energy and the resulting spacetime curvature (out of which the mirror is not part due to its inherently non-gravitational interaction with the neutron) and causal connection as the gravitational interaction propagates with the speed of light. However, as several experiments on entangled quantum states, starting with Clauser/Freedman [12] and Aspect et al. [13], seem to be compatible with a non-local connection between quantum objects [14], this property of the Schrödinger equation need not be a serious drawback for a theory of quantum gravity.

\footnotetext{
${ }^{5}$ In fact, a bound quantum gravitational object does not fall at all as it is described by a stationary wavefunction, or a superposition of such
} 
[1] M.B. Green, J.H. Schwarz \& E. Witten, Superstring Theory, 2 vol, Cambridge University Press (1987); J. Polchinski. String Theory, 2 vol, Cambridge University Press (1998).

[2] C. Rovelli, Quantum Gravity, (Cambridge University Press, 2004).

[3] R. Penrose, J.Math.Phys. 8, 345 (1967); Phys.Rep. 6, 241 (1972).

[4] A. Connes, Noncommutative Geometry, (Academic Press, 1994).

[5] C. Will, Theory and Experiment in Gravitational Physics, (Cambridge University Press, 1993).

[6] A.D. Sakharov Sov. Phys. Dokl. 12, 1040 (1968); Reprinted in Gen. Rel. Grav. 32, 365 (2000).

[7] J. Károlyházy, Nuovo Cim. A 42, 390 (1966).

[8] R. Penrose, Gravity and state vector reduction, in R. Penrose and C.J. Isham, editors, Quantum concepts in space and time, pages 129-146 (Clarendon Press, 1986).

[9] V.V. Nesvizhevsky et al., Nature 415, 297 (2002); Phys.Rev.D 67, 102002 (2003) hep-ph/0306198; Phys.Rev.D 68, 108702 (2003); J. Hansson et al., Phys.Rev.D 68, 108701 (2003) quant-ph/0308108

[10] T.F. Gallagher, Rydberg Atoms, (Cambridge University Press, 1994).

[11] J. Hansson, Acta Phys. Pol. B38, 91 (2007) astro-ph/0603342

[12] S.J. Freedman \& J.F. Clauser, Phys.Rev.Lett. 28, 938 (1972).

[13] A. Aspect, J. Dalibard \& G. Roger, Phys.Rev.Lett. 49, 91; 1804 (1982).

[14] J.S. Bell, Speakable and Unspeakable in Quantum Mechanics 2nd ed, (Cambridge University Press, 2004). 